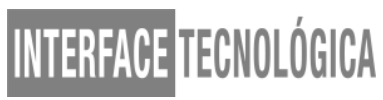

TECNOLOGIA BLOCKCHAIN: a disrupção na indústria financeira

\title{
BLOCKCHAIN TECHNOLOGY: the disruption in the financial industry
}

\author{
Júlio César de Miranda - paraojuliomiranda@gmail.com \\ Jederson Donizete Zuchi - jederson.zuchi@ fatec.sp.gov.br \\ Faculdade de Tecnologia de Taquaritinga (FATEC) - SP - Brasil
}

DOI: 10.31510/infa.v15i2.376

\begin{abstract}
RESUMO
Este artigo tem como objetivo apresentar a evolução histórica da tecnologia blockchain e seu surgimento como resposta à desconfiança no sistema financeiro centralizado durante a crise financeira de 2008, compreender os seus conceitos e funcionamento dentro do primeiro e maior blockchain, que é o Bitcoin, entender o conceito de hash dentro desta tecnologia, conhecer novas soluções de plataformas blockchain e principalmente investigar a revolução que esta tecnologia está gerando em vários setores, em especial na indústria de serviços financeiros. Para isso foi realizada uma pesquisa bibliográfica em fontes como livros, artigos, monografias, periódicos, revistas, simpósios, congressos, jornais e sites da internet. O blockchain apresenta infinitas possibilidades de aplicação, como resultado desta pesquisa percebe-se que setor financeiro antes ameaçado, investe grandes cifras em blockchain, na ordem de mais de US\$ 1 bilhão e está reinventando os seus processos e negócios, e especificamente aqui no Brasil, há poucos meses, o Banco Central e várias instituições financeiras desenvolveram protótipos e inovações em blockchain. Conclui-se que, blockchain é a tecnologia do "valor", é protagonista, revolucionária e disruptiva, tal como a World Wide Web nos anos de 1990, e a indústria financeira promete muitas inovações com esta tecnologia.
\end{abstract}

Palavras-chave: Blockchain. Bitcoin. Tecnologia. Criptografia. Financeiro.

\begin{abstract}
This article aims to present the historical evolution of blockchain technology and its emergence as a response to distrust in the centralized financial system during the 2008 financial crisis, to understand its concepts and functioning within the first and largest blockchain, which is Bitcoin, understand the hash concept within this technology, meet new solutions from blockchain platforms and mainly investigate the revolution that this technology is generating in various sectors, especially in the financial services industry. For this, a bibliographical research was carried out in sources such as books, articles, monographs, periodicals, magazines, symposia, congresses, newspapers and internet sites. The blockchain presents infinite possibilities of application, as a result of this research it is noticed that previously threatened financial sector, invests big numbers in blockchain, in the order of more than $\$ 1$ billion and is reinventing its processes and businesses, and specifically here in Brazil, a few months ago, the Central Bank and various financial institutions developed prototypes and innovations in blockchain. It is concluded that, blockchain is "value" technology, it is
\end{abstract}




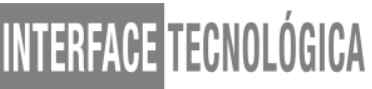

protagonist, revolutionary and disruptive, such as the World Wide Web in the 1990s, and the financial industry promises many innovations with this technology.

Keywords: Blockchain. Bitcoin. Technology. Cryptography. Financial.

\section{INTRODUÇÃO}

A capa do jornal Folha de São Paulo era "Maior quebra da história causa pior dia nas Bolsas desde o 11/09" (SCIARRETA, 2008). Um dia após a chamada Black Monday ou segunda-feira negra, este dia era 15 de setembro de 2008, o banco de investimentos Lehman Brothers anunciava que pediria concordata, conforme redação do Correio Braziliense (2009) o banco era considerado na época o $4^{\circ}$ maior dos Estados Unidos, este evento foi um terremoto global causando uma onda de desemprego e marcou a crise das hipotecas subprime (segunda linha), cujo gatilho foi em 08 de agosto de 2007 (POZZI, 2017).

De acordo com Franco e Bazan (2018), a causa da crise das hipotecas subprime, ou hipotecas podres, aconteceu anos antes, com a concessão sem controle de crédito imobiliário por parte dos bancos americanos para pessoas sem capacidade econômico financeira para a dívida, e com o preço em alta dos imóveis, as pessoas perceberam a vantagem de financiar o imóvel e depois vendê-lo, quitando a dívida e ainda obter um lucro.

Além disso investidores compravam títulos de crédito destas dívidas, classificados pelas agências de risco como baixo risco para investimento, quando veio a inadimplência dessas dívidas, os títulos de crédito lastreados nessas dívidas desses investidores viraram pó, sem receber o valor investido. Desencadeou a crise de desconfiança e de transparência do sistema financeiro, derrubando mercados globalmente e deteriorando a economia mundial.

Neste mesmo ano de 2008 de desconfiança mundial, de acordo com Tapscott e Tapscott (2016) e Franco e Bazan (2018), uma pessoa ou grupo de pessoas chamado de Satoshi Nakamoto publicou um artigo (paper) numa lista de discussão na internet, sobre um protocolo de transferência eletrônica de dinheiro entre as pessoas P2P (Peer-to-Peer) sem intermediário e descentralizado, ou seja, sem a figura da instituição financeira, este protocolo é o Bitcoin, em sua base está a tecnologia blockchain, ou como Tapscott e Tapscott (2016, p.38) diz "nós a estamos chamando de Protocolo da Confiança". A partir daí Satoshi Nakamoto abriu o debate sobre a confiança, ou melhor, a desconfiança em um sistema financeiro centralizado. 


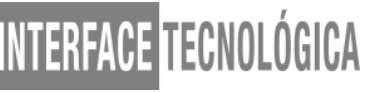

Diante desta contextualização, este artigo de caráter bibliográfico, tem por objetivo o estudo desta tecnologia disruptiva que é o blockchain, e que pode ser aplicada em qualquer indústria. A seção 2 apresenta a metodologia da pesquisa aplicada para o desenvolvimento do artigo, a seção 3 apresenta o conceito de blockchain e o seu funcionamento na rede do Bitcoin, a seção 4 trata das evoluções desta tecnologia, a partir do Bitcoin, como a plataforma Ethereum, e as plataformas Corda e Hyperledger que surgiram do esforço colaborativo de vários setores, incluindo o de serviços financeiros , e a apresentação de projetos blockchain que indústria financeira aqui no Brasil está desenvolvendo para o futuro dos seus negócios. A seção 5 apresenta as considerações finais.

\section{METODOLOGIA DE PESQUISA}

Para o desenvolvimento deste artigo foi realizada uma pesquisa bibliográfica em fontes como livros, artigos, monografias, periódicos, revistas, simpósios, congressos, jornais e sites da internet, e de acordo com Bovério e Silva (2018 apud PIZZANI et al. 2012, p. 54) definem a pesquisa bibliográfica como "a revisão da literatura sobre as principais teorias que norteiam o trabalho científico".

\section{CONCEITO DE BLOCKCHAIN E SEU FUNCIONAMENTO}

Um detalhe interessante é que o termo blockchain não é citado no artigo Bitcoin: A Peer-to-Peer Electronic Cash System, publicado em 2008 pela pessoa com o pseudônimo de Satoshi Nakamoto, ele explica que os registros históricos das movimentações são formados por uma série de blocos encadeados, onde cada bloco armazena as informações das transações com as criptomoedas bitcoins e dentro da rede Bitcoin, e nesta série, cada bloco é encadeado com criptografia ao bloco anterior, ou seja, uma cadeia de blocos, daí o termo blockchain. E de acordo com Franco e Bazan (2018) para a palavra bitcoin, recomenda-se a grafia inicial minúscula para a moeda e grafia inicial maiúscula para o protocolo.

Segundo Bovério e Silva (2018), para discorrer sobre a tecnologia blockchain é necessário compreender o funcionamento desta rede P2P do Bitcoin, pois, estão relacionadas. Nesse sentido Mougayar (2017, p. 4), afirma que "o blockchain é aquela invenção tecnológica por trás do Bitcoin, e o que o torna possível”. 


\section{WTEERFAET TECNOLLGGCA}

\subsection{Funcionamento e características do blockchain do Bitcoin}

Nakamoto (2008, p.1) descreve o protocolo Bitcoin como: "uma versão puramente peer-to-peer do dinheiro eletrônico permitiria que pagamentos on-line fossem enviados diretamente de uma parte para outra sem passar por uma instituição financeira”. E de acordo com Diniz (2017) a criptomoeda bitcoin, o dinheiro eletrônico desta rede foi lançada em janeiro de 2009 quando o protocolo Bitcoin entrou em funcionamento. A ilustração a seguir demonstra esta descrição e o funcionamento do blockchain do Bitcoin.

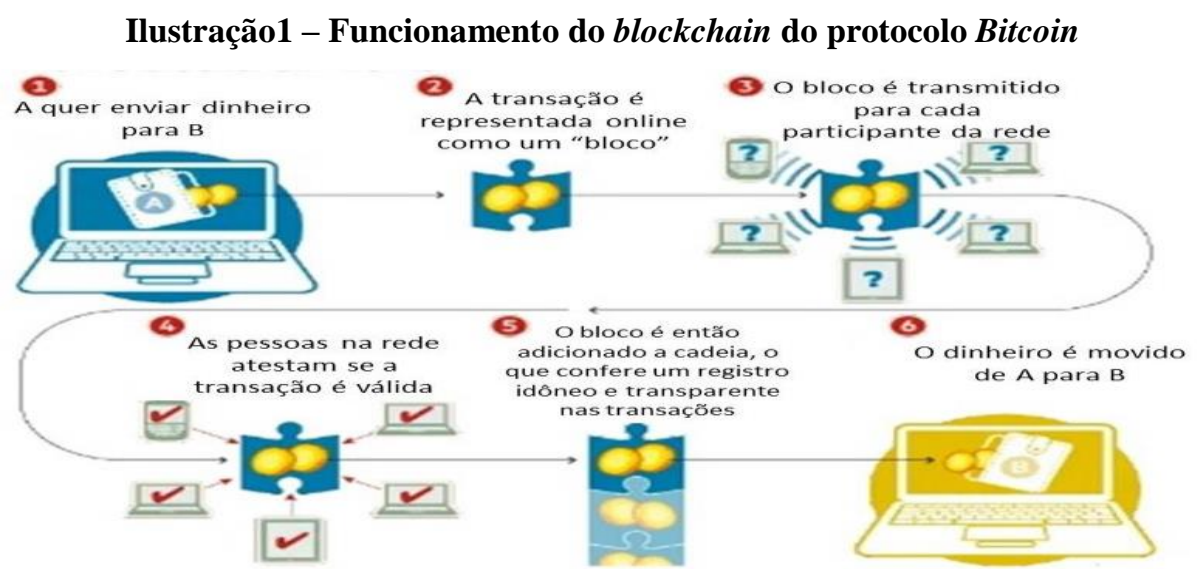

Fonte: Adaptado de Financial Times (WILD; ARNOLD; STAFFORD, 2015)

De acordo com Batista, Dias e Silva (2018), o blockchain funciona em uma rede distribuída de usuários, o que torna o registro confiável e idôneo, pois, todos da rede tem uma cópia deste registro para verificação das transações realizadas.

As pessoas atestam na rede se a transação é válida ou não, isso implica na quebra do paradigma de um validador único ou a regulação de um banco de dados central, a lógica é o consenso descentralizado (MOUGAYAR, 2017).

Segundo Araújo e Silva (2017, p.31) afirmam que "os registros online são carimbados com data e hora, não podem ser alterados pois é criptografado, a única forma de burlar seria conseguir o poder computacional maior que a rede inteira".

A tecnologia blockchain tem o mesmo entendimento para Katori (2017, p. 18) que diz "pode ser descrita como um sistema de registro cronológico".

Pires (2016, p. 26) complementa ao afirmar que "o blockchain é uma cadeia de registros imutáveis, públicos e distribuídos”. 
Verifica-se que os autores reafirmam que os conceitos de registro cronológico e imutável no blockchain, são uma segurança de dados e estão de acordo com os princípios citados por Nakamoto (2008), que são o servidor timestamp (que registra as transações em ordem cronológica) e a função hash. Franco e Bazan (2018) explicam que esta função é como uma via de sentido único ou também uma impressão digital, este algoritmo criptografa a mensagem, o que torna impossível sua reversão, pois, exige muita força computacional.

Desta forma, Diniz (2017) explica que Nakamoto propôs a solução do problema do double-spending, ou gasto duplo que é a utilização do mesmo valor ou unidade de pagamento em transações diferentes. Ou seja, se $\mathrm{X}$ transferir para $\mathrm{Y}$ um valor de $\mathrm{R} \$ 100,00$, este valor tem que sair de $\mathrm{X}$ e ir para $\mathrm{Y}$, ele não pode ser replicado pelo pagador, pois, o consenso descentralizado da rede e a criptografia garantem isso, contrapondo a confiança em uma autoridade central representada pelo sistema financeiro centralizado. Porém Nakamoto (2008, p. 1) observa que "o sistema é seguro desde que os nós honestos controlem coletivamente mais energia da CPU do que qualquer grupo colaborador de nós atacantes”. Essa ameaça na segurança da rede, Bovério e Silva (2018, p. 114) chamam de "ataque de 51 por cento".

De acordo com Batista, Dias e Silva (2018), estes nós que atestam e validam as transações são conhecidos como "mineradores", pois, eles gastam energia elétrica e poder computacional na resolução de problemas matemáticos complexos, ou seja, é uma prova para atestar a validade das transações, Nakamoto $(2008$, p. 3) chama este algoritmo de "Prova de Trabalho", (do inglês Proof-of-Work ou PoW), quando esta prova é resolvida, os nós da rede recebem as moedas digitais bitcoins, como incentivo ao suporte ao funcionamento da rede, estimulando a honestidade dos nós e assim minimizar o risco do "ataque de 51 por cento". Nakamoto $(2008$, p. 4) compara os nós que trabalham nesta prova aos "mineradores de ouro que gastam recursos para adicionar ouro à circulação".

Segundo Franco e Bazan (2018), a recompensa do processo de mineração do bitcoin, tem redução de $50 \%$ a cada intervalo de 4 anos, hoje está 12,5 bitcoins por bloco encontrado, o próximo reajuste será em 2020. Com o passar do tempo dessa mineração, a criptomoeda fica mais escassa, até atingir o limite de 21 milhões de moedas produzidas, a expectativa em 2032, é que mais de $99 \%$ deste limite tenha sido minerado. Muitos mineradores se juntam em locais virtuais chamados de pools de mineração, para compartilhamento de hashrate (poder computacional) para mineração das criptomoedas.

Ainda segundo Rickards (2018), alguns mineradores de bitcoins que possuem servidores com alto poder de processamento, estão localizados na Islândia por ser um país frio 
que compensa o calor gerado pelo processamento, e com sistema de energia geotérmica, o que torna a energia elétrica barata.

\subsection{O conceito de hash no blockchain}

O bloco no blockchain é identificado por um hash, que é uma mensagem criptografada, resultado de uma função hash criptográfica, que é um algoritmo computacional de criptografia. Segundo Narciso (2018, p. 323) diz que "geração de um hash, o qual também é conhecido como um checksum ou assinatura digital, é um dado que resulta do cálculo feito por um algoritmo de criptografia (no caso o SHA256), aplicado a um determinado arquivo". Ainda de acordo com o autor, não importa o tamanho da mensagem, "o hash gerado é sempre com o mesmo comprimento", ou seja, uma cadeia hexadecimal de 64 dígitos. Na tabela 1 a seguir, temos o exemplo de hash.

Tabela 1 - Exemplos de hashes

\begin{tabular}{c|c}
\hline O hash da palavra "Blockchain" com a letra & $\begin{array}{c}\text { 625da44e4eaf58d61cf048d168aa6f5e } \\
\text { 492dea166d8bb54ec06c30de07db57e1 }\end{array}$ \\
\hline B maiúsculo & ef7797e13d3a75526946a3bcf00daec \\
minúscula & 9fc9c9c4d51ddc7cc5df888f74dd434d1 \\
\hline
\end{tabular}

Fonte: Adaptado de Narciso (2018, p. 324)

O hash é uma assinatura digital de um bloco, esta é usada para encadeá-lo ao bloco anterior, e este ligado ao seu bloco antecessor e assim por diante até o primeiro bloco desta cadeia. O bloco do blockchain armazena várias transações e de acordo com Franco e Bazan (2018), ele possui duas partes que são o cabeçalho e a lista de transações. Neste cabeçalho fica a identificação do bloco, no caso o seu hash, conforme demonstra a ilustração seguinte.

Ilustração 2 - Ligação de hashes da cadeia de blocos

\begin{tabular}{|c|c|c|c|}
\hline & Bloco 60 & Bloco 61 & Bloco 62 \\
\hline Prova de Trabalho & \begin{tabular}{|l} 
a7faf89gb90 \\
\end{tabular} & iasm91hnv8 & $1 \mathrm{k} 3 g n c h g O u$ \\
\hline Bloco Anterior & ug90bsdg98 & a7faf89gb90 & iasm91hnv8 \\
\hline Transação 1 & 285412 & b54a80 & cb54a8 \\
\hline Transação 2 & a9d347 & $25569 d$ & a808a2 \\
\hline Transação 3 & $2 \mathrm{e} 970 \mathrm{c}$ & a47919 & f810d4 \\
\hline
\end{tabular}

Fonte: Extraído de Lima, Hitomi e Oliveira (2018, p. 8) 
Franco e Bazan (2018) explicam que o hash de um bloco é o resultado de um cálculo do hash do bloco antecessor e assim por diante, os autores afirmam que após a adição de seis blocos à frente de um certo bloco, dado à complexidade matemática da criptografia, a tentativa de fraudar esta estrutura do blockchain é inviável, fica mais segura com o tempo.

\section{BLOCKCHAIN: A REVOLUÇÃO DEPOIS DA INTERNET}

Apresentados os conceitos do blockchain e do protocolo do Bitcoin, que atualmente é o maior blockchain em funcionamento, é notável que após a criação do blockchain do Bitcoin, nestes últimos 10 anos, o termo blockchain é constantemente citado na mídia, muitas reportagens, em artigos técnicos e científicos, pode-se chamar de uma tecnologia disruptiva tal como foi a Internet no século passado, nos anos de 1990. Tapscott e Tapscott (2017, p. 21) afirma que "se a internet foi o primeiro formato digital nativo da informação, então o blockchain é o primeiro formato digital nativo do valor - o novo meio para o dinheiro".

Em outra obra de Tapscott e Tapscott (2016) ele afirma que o blockchain é o novo livro-razão digital, tudo o que for de valor e importante pode ser gravado no "blockchain", de documentos como certidões de nascimento a títulos de propriedade, indenizações de seguro, rastreamento na produção na cadeia de alimentos, votos, uma infinidade de valores que podem ser programados e registrados nesta tecnologia.

Diniz (2017, p. 49) afirma que "de cartórios a sistemas de registros de transações comerciais, muitas atividades estão sendo ameaçadas pelo potencial disruptivo do blockchain". Sobre esta disrupção, o autor cita o termo "Blockchain 2.0" para as aplicações em blockchain em outras funcionalidades que não seja meios de pagamento ("Blockchain 1.0”). Segundo Bovério e Silva (2018 apud SWAN, 2015) as gerações do blockchain é divido em três, sendo o Blockchain 1.0 referente à descentralização do dinheiro, a segunda geração que é Blockchain 2.0, a descentralização dos mercados de forma geral. E a terceira geração, o Blockchain 3.0, voltado à pesquisas e projetos nas áreas governamentais.

No entanto, de acordo com Bovério e Silva (2018 apud GATES, 2017) reafirmam com Diniz (2017) a divisão das gerações do blockchain em duas, onde a terceira e a segunda geração são uma só, o Blockchain 2.0 é marcado com o surgimento em janeiro de 2014 do blockchain do Ethereum de código aberto tal como o Bitcoin, fundado por Vitalik Buterin, é uma plataforma cujo objetivo é ser um imenso computador descentralizado que permita 
processar qualquer aplicação na rede, inclusive do smart contract, contrato inteligente que funciona sem a necessidade de um intermediário que garanta seu cumprimento.

Mougayar (2017) afirma que os contratos inteligentes representam um poder revolucionário no blockchain, podendo substituir funções de intermediários burocráticos e caros, como por exemplo o registro de títulos de propriedade, entre outras aplicações.

E com toda esta revolução que o blockchain está propondo em todos os setores, a indústria de serviços financeiros vem ganhando destaque no desenvolvimento e implementação desta tecnologia aos seus negócios, o que antes era uma ameaça quando do surgimento do protocolo do Bitcoin, hoje esta tecnologia representa uma oportunidade para reconstrução de seus processos e redução de seus custos, e segundo Tapscott e Tapscott (2016, p.39) diz que "a indústria de serviços financeiros já rebatizou e privatizou a tecnologia blockchain, referindo-se a ela como tecnologia de contabilidade distribuida".

A Deloitte (uma das maiores empresas de consultoria do mundo) em conjunto com o Fórum Econômico Mundial publicou um relatório que constatou, que há mais de 2500 pedidos de patente de DLT (Distributed Ledger Technology) ou tecnologia de livro-razão distribuído pela indústria financeira, e o setor acumula mais de US\$ 1,4 bilhão em investimentos em apenas três anos (BLOCKCHAIN, 2017).

\subsection{As plataformas Corda e Hyperledger}

A plataforma blockchain Corda foi desenvolvida com código aberto pelo consórcio R3, empresa de tecnologia sediada em Nova Iorque, onde neste grupo participa mais de 70 instituições financeiras do mundo (KELLY, 2016).

Segundo Ferreira (2017) a plataforma Corda foi projetada para atender as necessidades do setor financeiro, tendo como funcionalidades: gerenciamento de transações entre duas partes, uso de ferramentas do padrão do setor financeiro, suporta vários mecanismos de consenso e restrição de acesso aos dados dentro de um contrato, exceto para quem tem direito.

A plataforma blockchain Hyperledger de código aberto, iniciado em 2015 pela Linux Foundation é um projeto colaborativo que envolve várias indústrias do setor financeiro, tecnologia e logística, cujo objetivo é suportar várias aplicações em blockchain. De acordo com Ferreira (2017) entre as características desta plataforma são: o gerenciamento das identidades dos usuários, privacidade nas transações confidenciais e que coexistam na mesma rede de permissões, e eficiência, onde cada nó processa várias transações simultaneamente. 


\subsection{Os desenvolvimentos de aplicações blockchain na indústria financeira brasileira}

De acordo com Faustino (2018), o grupo Blockchain Febraban (Federação Brasileira de Bancos) criado em 2017 para pesquisa e desenvolvimento em blockchain, é formado por 18 bancos e instituições do setor, apresentaram no evento CIAB 2018 (congresso) realizado de 12 a 14 de junho de 2018 em São Paulo, um protótipo blockchain para compartilhar informações de segurança sobre dispositivos móveis que venham a ser usados pelos clientes na sua relação com o banco, essas informações alimentam os sistemas antifraude para constatar se um celular foi roubado, perdido ou furtado. A plataforma escolhida para esta operação foi o Hyperledger Fabric.

Também recentemente, o Banco Central do Brasil (2018) anunciou em seu site, a plataforma PIER (Plataforma de Integração de Informações das Entidades Reguladoras) em blockchain para troca de dados com órgãos reguladores do sistema financeiro.

Outra iniciativa no setor bancário em blockchain, talvez a mais interessante, de acordo com Temóteo (2018), foi anunciado no $11^{\circ}$ Fórum Internacional de Tecnologia da Informação do Banrisul (realizado de 23 a 24 de maio de 2018), o sistema SFD (Sistema Financeiro Digital) que funciona 24 horas por dia, é um serviço baseado em blockchain, desenvolvido pelos bancos Banco do Brasil, Caixa Econômica Federal, Sicoob, Banrisul e Santander, o usuário fará transferências seguras apenas selecionando o contato da agenda telefônica do celular, um serviço P2P sem passar por uma custódia de uma terceira parte.

O SFD é uma alternativa às transferências via DOC (documento de crédito) que demora até 3 dias úteis para compensação ou via TED (transferência eletrônica disponível) que é liquidada em minutos no mesmo dia, porém ambas funcionam somente em dias úteis. $\mathrm{O}$ projeto piloto do SFD será iniciado nos próximos meses, em seguida estará disponível aos clientes destes bancos

Essa inovação cria uma expectativa muito grande na indústria financeira, para a criação de um sistema rápido de ordens de pagamento e transferências bancárias internacionais baseado em blockchain, pois, os meios atuais demoram alguns dias para liquidação em razão da centralização em uma terceira parte como a rede do Western Union e do SWIFT (sigla em inglês que significa Sociedade de Telecomunicações Financeiras Interbancárias Mundiais), o que contribuiria para a redução de custos tanto para os clientes usuários, bem como para as próprias instituições financeiras. 


\section{CONSIDERAÇÕES FINAIS}

Ao final deste artigo, há uma constatação, do quão disruptiva é a tecnologia blockchain, que quando criada junto com o Bitcoin em meio à crise econômica de 2008, se apresentou como rival ao sistema financeiro centralizado, e que com o passar dos anos, e com o melhor entendimento desta tecnologia, ainda considerada jovem, apresenta infinitas possibilidades de aplicações ao nosso mundo, tão revolucionária quanto à Web nos anos de 1990, e o mais interessante, é que a indústria financeira está desenvolvendo esta tecnologia de uma forma sem igual. E nos últimos 10 anos, o crescente interesse pela tecnologia blockchain é exponencial, o que produziu muito material para pesquisa e desenvolvimento, fato que se comprova com o extenso material acadêmico como livros, artigos, sites, entre outros.

A tecnologia blockchain tem muita inovação a proporcionar e muitos capítulos estão por vir com novas descobertas e funcionalidades para a humanidade.

\section{REFERÊNCIAS}

ARAÚJO, H. P.; SILVA, R. B. A. R. A tecnologia digital blockchain: análise evolutiva e pragmática. REFAS - Revista Fatec Zona Sul, v.3, n.4, p 23-39, jun. 2017. Disponível em: < http://www.revistarefas.com.br/index.php/RevFATECZS/article/view/98/118>. Acesso em: 07 ago. 2018.

BANCO CENTRAL DO BRASIL. Plataforma do BC com tecnologia blockchain facilitará troca de dados na supervisão do sistema financeiro. Brasília, 11 abr. 2018. Disponível em: <https://www.bcb.gov.br/pt-br/\#!/c/noticias/249>. Acesso em 04 ago. 2018.

BATISTA, A. O. A.; DIAS, E. R. B.; SILVA, M. B; ROCHA, C. (Org). Identificação digital baseada em blockchain: Um conceito disruptivo no ciberespaço. In: Simpósio Internacional de Inovação em Mídias Interativas, 5., 2018, Goiânia. Anais do V Simpósio Internacional de Inovação em Mídias Interativas, p. 307-320. Goiânia: Media Lab / UFG, 2018. ISSN 2358-0488. Disponível em: 〈https://siimi.medialab.ufg.br/up/777/o/28_-_Alex_Batista.pdf〉. Acesso em: 07 ago. 2018.

BLOCKCHAIN e o futuro da infraestrutura financeira. Deloitte, São Paulo, p. 1-21, 2017. Disponível em: <https://www2.deloitte.com/br/pt/pages/finance/articles/blockchain-servicosfinanceiros.html\#>. Acesso em: 07 ago. 2018.

BOVÉRIO, M. A.; SILVA, V. A. F. BLOCKCHAIN. Revista Interface Tecnológica, v. 15, n. 1, p. 109-121, 30 jun. 2018. Disponível em: <http://revista.fatectq.edu.br/index.php/interfacetecnologica/article/view/326/217>. Acesso em: 25 jul. 2018. 
CORREIO BRAZILIENSE. 15 de setembro de 2008: quebra do Lehman Brothers dá início à crise que abalou o mundo. Brasília, 15 set. 2009. Disponível em:

<https://www.correiobraziliense.com.br/app/noticia/economia/2009/09/15/internas_economia, 142192/15-de-setembro-de-2008-quebra-do-lehman-brothers-da-inicio-a-crise-que-abalou-omundo.shtml>. Acesso em: 07 ago. 2018.

DINIZ, E. H. Emerge uma nova tecnologia disruptiva. Revista GV-Executivo, São Paulo, v. 16, n. 2, p. 46-50, mar/abr. 2017. Disponível em:

<http://bibliotecadigital.fgv.br/ojs/index.php/gvexecutivo/article/view/68676>. Acesso em: 07 ago. 2018.

FAUSTINO, R. Febraban cria solução em blockchain para verificar segurança de dispositivos móveis. Revista Época Negócios, Rio de Janeiro, 13 jun. 2018. Disponível em:

$<$ https://epocanegocios.globo.com/Tecnologia/noticia/2018/06/febraban-cria-solucao-em-

blockchain-para-verificar-seguranca-de-dispositivos-moveis.html>. Acesso em: 04 ago. 2018.

FERREIRA, J. E. Blockchain para criação de novos modelos de negócio e seus impactos na indústria de serviços financeiros. 2017. 51f. Trabalho de Graduação (Sistemas de Informação), Universidade Federal de Pernambuco, Recife, 2017. Disponível em: <http://www.cin.ufpe.br/ tg/2017-1/jef-tg.pdf>. Acesso em: 07 ago. 2018.

FRANCO, A.; BAZAN, V. Criptomoedas: melhor que dinheiro. Prefácio: Safiri Felix. São Paulo: Empiricus, 2018. 208p.

GATES, M. Blockchain: Ultimate Guide to Understanding Blockchain, Bitcoin, Cryptocurrencies, Smart Contracts and the Future of Money. Breinigsville, Pensilvânia: Createspace Independent Publishing Platform. 2017. 126 p.

KATORI, F. Y. Impacto das fintechs e do blockchain no sistema financeiro: uma análise crítico-reflexiva. 2017. 33f., il. Trabalho de Conclusão de Curso (Bacharelado em Ciências Contábeis), Universidade de Brasília, Brasília, 2017. Disponível em:

<http://bdm.unb.br/handle/10483/19517>. Acesso em: 07 ago. 2018.

KELLY, J. Plataforma blockchain desenvolvida por bancos terá código aberto. Reuters, Londres, 20 out. 2016. Disponível em:

<https://br.reuters.com/article/internetNews/idBRKCN12K2F7>. Acesso em: 08 ago. 2018.

LIMA, B. H. N.; HITOMI, F. A. C.; OLIVEIRA, G. S. Aplicação da tecnologia blockchain em ambientes corporativos. FaSCI-Tech, v. 1, n. 13, p. 6-13, jun. 2018. Disponível em: <http://www.fatecsaocaetano.edu.br/fascitech/index.php/fascitech/article/view/133>. Acesso em: 14 ago. 2018.

MOUGAYAR, W. Blockchain para negócios: promessa, prática e aplicação da nova tecnologia da internet. Tradução: Vivian Sbravatti. Rio de Janeiro: Alta Books, 2017. 224p.

NAKAMOTO, S. Bitcoin: A Peer-to-Peer Electronic Cash System. Disponível em: $<$ https://bitcoin.org/bitcoin.pdf>. Acesso em: 07 ago. 2018. 
NARCISO, P. H. D.; ROCHA, C. (Org). Blockchain como garantia de direitos autorais. In: Simpósio Internacional de Inovação em Mídias Interativas, 5., 2018, Goiânia. Anais do V Simpósio Internacional de Inovação em Mídias Interativas, p. 321-325. Goiânia: Media Lab / UFG, 2018. ISSN 2358-0488. Disponível em: <https://siimi.medialab.ufg.br/up/777/o/29_-_Paulo_Narciso.pdf>. Acesso em: 07 ago. 2018

PIRES, T. P. Tecnologia blockchain e suas aplicações para provimento de transparência em transações eletrônicas. 2016. 57 f., il. Trabalho de Conclusão de Curso (Bacharelado em Engenharia de Redes de Comunicação), Universidade de Brasília, Brasília, 2016. Disponível em: <http://bdm.unb.br/handle/10483/16252>. Acesso em: 07 ago. 2018.

PIZZANI, L. et al. A arte da pesquisa bibliográfica na busca do conhecimento. In: Revista Digital de Biblioteconomia e Ciência da Informação, Campinas, São Paulo, v.10, n. 1, p.5366, jul. 2012. Disponível em:

<https://periodicos.sbu.unicamp.br/ojs/index.php/rdbci/article/view/1896>. Acesso em: 07 ago. 2018.

POZZI, S. Bolha imobiliária: dez anos do gatilho da crise que parou o mundo. El País, Nova York, 07 ago. 2017. Disponível em:

<https://brasil.elpais.com/brasil/2017/08/05/economia/1501927439_342599.html>. Acesso em: 07 ago. 2018.

RICKARDS, J. Cripto wars: a guerra por trás das criptomoedas. Tradução: Mahana Pelosi. Prefácio: Shae Russel. São Paulo: Empiricus, 2018. 192 p.

SCIARRETA, T. Bolsas têm o pior dia desde o 11 de Setembro. Folha de São Paulo, São Paulo, 16 set. 2008. Disponível em:

<https://www1.folha.uol.com.br/fsp/dinheiro/fi1609200802.htm>. Acesso em: 07 ago. 2018.

SWAN, M. Blockchain: Blueprint for a New Economy. Sebastopol, California: O'Reilly Media Inc., 2015. 149 p.

TAPSCOTT, D.; TAPSCOTT, A. A revolução do blockchain: transformando os alicerces dos serviços financeiros. Resenha B3, São Paulo, n.6, p. 20-24, ago. 2017. Disponível em: <http://resenhab3.com.br/wp-content/uploads/2017/08/ResenhaB3_ED6-Internet.pdf>. Acesso em: 07 ago. 2018.

TAPSCOTT, D.; TAPSCOTT, A. Blockchain Revolution: como a tecnologia por trás do Bitcoin está mudando o dinheiro, os negócios e o mundo. São Paulo: SENAI - SP Editora, 2016. 392 p.

TEMÓTEO, A. Bancos criam serviço de transferência 24 horas baseado em blockchain. Correio Braziliense, Brasília, 23 mai. 2018. Disponível em:

$<$ http://blogs.correiobraziliense.com.br/vicente/bancos-criam-servico-de-transferencia-24horas-baseado-em-blockchain/>. Acesso em: 04 ago. 2018.

WILD, J.; ARNOLD, M.; STAFFORD, P. Technology: Banks seek the key to blockchain. Financial Times, 01 nov. 2015. The Big Read, Clearing \& Settlement. Disponível em: 


\section{|WTERFFACETECENOLGGCA}

<https://www.ft.com/content/eb1f8256-7b4b-11e5-a1fe-567b37f80b64>. Acesso em: 08 ago. 2018. 